Pacific Journal of Mathematics

GLOBAL ALMOST ANALYTIC ALGEBRAICITY OF ANALYTIC
SETS 


\title{
GLOBAL ALMOST ANALYTIC ALGEBRAICITY OF ANALYTIC SETS
}

\section{WOJCIECH KUCHARZ}

\begin{abstract}
Let $X$ be a nonsingular real algebraic subset of $\mathbf{R}^{n}$. There are known several results concerning analytic subsets of $X$ which are analytically or $C^{r}$ equivalent to algebraic sets, i.e., which can be transformed by an analytic or $C^{r}$ diffeomorphism, $r=0,1,2, \ldots$, of $X$ onto algebraic subsets. In general, $C^{r}$ equivalence does not imply analytic equivalence. however, we show in this paper that $C^{r}$ equivalence often can be replaced by, much stronger, "almost analytic" equivalence.
\end{abstract}

1. The main result. In this paper real algebraic varieties and morphisms between them are understood in the sense of Serre [15] (Serre considers algebraic varieties over an algebraically closed field but his basic definitions make sense over any field). The reader may consult a detailed exposition [7] for properties of real algebraic varieties, especially in connection with real algebraic blow-ups (cf. also [1]). All subvarieties will be assumed closed but not necessarily irreducible.

Let $Y$ be an affine real algebraic variety and let $Z$ be a subvariety of $Y$. Then the algebraic blow-up $\pi: B \rightarrow Y$ of $Y$ along $Z$ has the following properties: $B$ is an affine real algebraic variety, $\pi$ is a real algebraic morphism whose restriction to $B \backslash \pi^{-1}(Z)$ is an algebraic isomorphism onto $Y \backslash Z$, and $\pi$ is a proper map if $B$ and $Y$ are equipped with the Euclidean topology. Moreover, $B$ is nonsingular and $\pi$ is surjective if $Y$ and $Z$ are nonsingular varieties.

Let $\widetilde{X}$ and $X$ be affine nonsingular real algebraic varieties and let $D$ be a subvariety of $X$. An algebraic morphism $\pi: \widetilde{X} \rightarrow X$ is called a $k$-fold algebraic multiblowup of $X$ along $D$ if $\pi$ is the composition $\pi=\pi_{1} \circ \cdots \circ \pi_{k}$, where

$$
\tilde{X}=X_{k} \stackrel{\pi_{k}}{\longrightarrow} X_{k-1} \stackrel{\pi_{k-1}}{\longrightarrow} \cdots \stackrel{\pi_{2}}{\longrightarrow} X_{1} \stackrel{\pi_{1}}{\longrightarrow} X_{0}=X,
$$

$\pi_{1}$ is the algebraic blow-up of $X_{0}$ along a nonsingular subvariety of $D$ and $\pi_{i+1}$ is the algebraic blow-up of $X_{i}$ along a nonsingular subvariety of $X_{i}$ contained in $\left(\pi_{1} \circ \ldots \circ \pi_{i}\right)^{-1}(D)$ for $i=1, \ldots, k-1$. Note that the restriction of $\pi$ to $\widetilde{X} \backslash \pi^{-1}(D)$ is an algebraic isomorphism onto $X \backslash D$. 
To formulate the main result, we need one more notion.

Given a compact (in the Euclidean topology) affine real algebraic variety $X$ and a nonnegative integer $k$, we denote by $H_{k}^{\text {alg }}(X, \mathbf{Z} / 2)$ the subgroup of the homology group $H_{k}(X, \mathrm{Z} / 2)$ generated by the homology classes represented by the algebraic $k$-dimensional subvarieties of $X$ (cf. [7], Chap. 11 or [8]). If $V$ is a $k$-dimensional analytic subset of $X$, then $[V]$ will stand for the homology class in $H_{k}(X, \mathbf{Z} / 2)$ represented by $V[8]$.

THEOREM 1.1. Let $X$ be a compact affine nonsingular real algebraic variety of dimension $n$. Let $V_{1}, \ldots, V_{d}$ be coherent analytic subsets of $X$. Assume that there exists a finite subset $D$ of $X$ such that $V_{i} \backslash D$ is an analytic submanifold of $X$ of codimension 1 for $i=1, \ldots, d$ and the submanifolds $\left\{V_{i} \backslash D\right\}_{i=1, \ldots, d}$ are in general position. Also assume that $\left[V_{i}\right]$ belongs to $H_{n-1}^{\mathrm{alg}}(X, \mathrm{Z} / 2)$ for all $i=1, \ldots, d$. Then there exists an algebraic multiblowup $\pi: \widetilde{X} \rightarrow X$ of $X$ along $D$ with the property that for each nonnegative integer $r$, one can find a $C^{r}$ diffeomorphism $\sigma: X \rightarrow X$ and an analytic diffeomorphism $\tilde{\sigma}: \widetilde{X} \rightarrow \widetilde{X}$ such that $\pi \circ \tilde{\sigma}=\sigma \circ \pi$ and $\sigma\left(V_{i}\right)$ is an algebraic subvariety of $X$ for $i=1, \ldots, d$.

Of course, "general position" in Theorem 1.1 has the usual meaning (cf. for instance [6], p. 2).

In the present work we adopt the point of view suggested by $\mathrm{T}$. C. Kuo (cf. [11], [12], [13], [14] and also [9]) who searched for equivalence relations weaker than analytic equivalence but still preserving its nice features. Note that, in Theorem 1.1, $\sigma \mid X \backslash D$ is analytic. Moreover, $\sigma$ is "almost analytic" at $D$ in the sense that the obstruction which prevents it from being analytic at $D$ is of a very special nature. Namely, $\sigma$ can be "lifted" to an analytic diffeomorphism $\tilde{\sigma}$ of $\widetilde{X}$. Observe also that the analytic subsets $\pi^{-1}\left(V_{i}\right)$ of $\tilde{X}, i=1, \ldots, d$, can be simultaneously analytically transformed onto algebraic subvarieties of $\widetilde{X}$. More precisely, $\tilde{\sigma}\left(\pi^{-1}\left(V_{i}\right)\right), i=1, \ldots, d$, are algebraic subvarieties of $\widetilde{X}$. We should also mention that $\sigma$ cannot, in general, be chosen analytic (cf. [5], p. 118, Example 3).

Theorem 1.1 immediately implies the following corollary.

COROLlary 1.2. If $V$ is a coherent analytic subset of $X$ such that $V$ is of pure dimension $n-1$ at each point, the set of singular points $\operatorname{Sign}(V)$ of $V$ is finite, and $[V]$ belongs to $H_{n-1}^{\text {alg }}(X, \mathbf{Z} / 2)$, then the 
conclusion of Theorem 1.1 holds true with $d=1, V_{1}=V$ and $D=$ $\operatorname{Sing}(V)$.

REMARK 1.3. The assumptions " $\left[V_{i}\right]$ belongs to $H_{n-1}^{\text {alg }}(X, \mathbf{Z} / 2)$ " in Theorem 1.1 and " $[V]$ belongs to $H_{n-1}^{\text {alg }}(X, \mathbf{Z} / 2)$ " in Corollary 1.2 cannot be dropped (cf. [5], p. 116, Example 1).

2. The key proposition. We start by recalling the following important result.

LEMMA 2.1. Let $Y$ be a closed analytic submanifold of an analytic manifold $X$ and let $\pi: B \rightarrow X$ be the analytic blow-up of $X$ along $Y$. Let $\nu$ be an analytic vector field on $X$ which is $k$-flat at $Y$ for some positive integer $k$. Then the vector field $\nu^{*}$ on $B$ defined by

$$
\nu^{*}(p)= \begin{cases}\left(d_{p} \pi\right)^{-1}(\nu(\pi(p))) & \text { for } p \in B \backslash \pi^{-1}(Y), \\ 0 & \text { for } p \in \pi^{-1}(Y)\end{cases}
$$

is analytic and $(k-1)$-flat at $\pi^{-1}(Y)$. If $\nu$ analytically depends on a parameter, then $\nu^{*}$ also analytically depends on the parameter.

Proof. This result, which is well known, is of a local nature and follows from a straightforward calculation in local coordinates.

Every manifold considered below will be equipped, even if we do not explicitly say so, with a fixed Riemannian metric. If $M$ is a manifold, $x$ is a point in $M$ and $D$ is a subset of $M$, then $\operatorname{dist}(x, D)$ will denote the distance from $x$ to $D$ with respect to the fixed Riemannian metric on $M$.

Our next auxiliary result is a consequence of Hironaka's desingularization theorem.

LEMMA 2.2. Let $X$ be a compact affine nonsingular real algebraic variety and let $f: X \rightarrow \mathbf{R}$ be an analytic function with $f^{-1}(0)=D$ finite. Then there exists an algebraic multiblowup $\pi: \tilde{X} \rightarrow X$ of $X$ along $D$ such that $f \circ \pi$ is locally a normal crossing, i.e., each point $p$ in $\tilde{X}$ has a local analytic coordinate system $\left(U_{p}, y_{1}, \ldots, y_{n}\right)$ with origin at $p$ such that $f \circ \pi \mid U_{p}=\varepsilon y_{1}^{\mu(1)} \cdots y_{n}^{\mu(n)}$, where $\varepsilon: U_{p} \rightarrow \mathbf{R}$ is a nowhere vanishing analytic function and $\mu(1), \ldots, \mu(n)$ are nonnegative integers.

Proof. By the Lojasiewicz inequality [16],

$$
|f(x)| \geq a \operatorname{dist}(x, D)^{m} \text { for all } x \text { in } X,
$$


where $a>0$ and $m$ is a positive integer. Let $\varphi: X \rightarrow \mathbf{R}$ be a regular function close to $f$ in the $C^{\infty}$ topology, such that $f-\varphi$ is $m$-flat at $D$ (cf. [4], Corollary 1). We may assume that $\varphi^{-1}(0)=D$. By Hironaka's desingularization theorem [10], there exists an algebraic multiblowup $\pi: \widetilde{X} \rightarrow X$ of $X$ along $D$ such that $\varphi \circ \pi$ is locally a normal crossing.

We claim that $f \circ \pi$ is also locally a normal crossing. Indeed, let $p$ be a point in $\tilde{X}$ and let $\left(U_{p}, y_{1}, \ldots, y_{n}\right)$ be an analytic local coordinate system on $\widetilde{X}$ with center at $p$ such that $\varphi \circ \pi \mid U_{p}=$ $\delta y_{1}^{\mu(1)} \cdots y_{n}^{\mu(n)}$, where $\delta: U_{p} \rightarrow \mathbf{R}$ is an analytic nowhere vanishing function and $\mu(1), \ldots, \mu(n)$ are nonnegative integers. Since $f$ satisfies (2.2.1) and $f-\varphi$ is $m$-flat at $D$, we obtain that for every point $x$ in $X$ and every analytic map-germ $\gamma:(\mathbf{R}, 0) \rightarrow(X, x)$, both $f \circ \gamma$ and $\varphi \circ \gamma$ have the same order of flatness at 0 . Clearly, the analogous observation is valid for $f \circ \pi \circ \tilde{\gamma}$ and $\varphi \circ \pi \circ \tilde{\gamma}$, where $\tilde{\gamma}:(\mathbf{R}, 0) \rightarrow(\widetilde{X}, q)$ is an analytic map-germ, $q \in \tilde{X}$. It follows that $f \circ \pi \mid U_{p}=\varepsilon Y_{1}^{\mu(1)} \cdots y_{n}^{\mu(n)}$ for some analytic nowhere vanishing function $\varepsilon: U_{p} \rightarrow \mathbf{R}$. Hence, $f \circ \pi$ is locally a normal crossing and the lemma is proved.

For $f, \pi$ and $\left(U_{p}, y_{1}, \ldots, y_{n}\right)$ as in Lemma 2.2 , the integer $h(f, \pi, p)=\max \{\mu(1), \ldots, \mu(n)\}$ does not depend on the choice of $\left(U_{p}, y_{1}, \ldots, y_{n}\right)$. Since $\pi^{-1}(D)$ is compact,

$$
h(f, \pi)=\sup \{h(f, \pi, p) \mid p \in X\}
$$

is finite. The integer $h(f, \pi)$ will play an important role later on.

Given a map $F: X \times[0,1] \rightarrow Y$ and a point $t$ in $[0,1]$, let $F_{t}$ denote the map $F(\cdot, t)$.

LEMMA 2.3. Let $X$ be an affine nonsingular real algebraic variety and let $\pi: \widetilde{X} \rightarrow X$ be an algebraic $k$-fold multiblowup of $X$ along $a$ finite subset $D$ of $X$. Let $f: X \times[0,1] \rightarrow \mathbf{R}$ and $\tilde{g}: \widetilde{X} \times[0,1] \rightarrow \mathbf{R}$ be analytic functions. Assume that $f_{0} \circ \pi$ is locally a normal crossing and

$$
f(x, t) \geq a \operatorname{dist}(x, D)^{m} \text { for all }(x, t) \text { in } X \times[0,1],
$$

where $a>0$ and $m$ is a positive integer. Also assume that $f_{t}^{-1}(0)=$ $D, f_{t}-f_{0}$ is $m$-flat at $D$, and $\tilde{g}_{t}$ is $h\left(f_{0}, \pi\right)$-flat at $\pi^{-1}(0)$ for all $t$ in $[0,1]$. Then there exists an analytic function $\tilde{h}: \tilde{X} \times[0,1] \rightarrow \mathbf{R}$ which vanishes on $\pi^{-1}(D) \times[0,1]$ and satisfies $\tilde{h}(p, t) f(\pi(p), t)=\tilde{g}(p, t)$ for $(p, t)$ in $\widetilde{X} \times[0,1]$. 
Proof. As in the proof of Lemma 2.2, one easily sees that $f(\pi(p), t)$ $=\tilde{u}(p, t) f_{0}(\pi(p))$, where $\tilde{u}: \tilde{X} \times[0,1] \rightarrow \mathbf{R}$ is a nowhere vanishing analytic function. The proof is concluded since the $h\left(f_{0}, \pi\right)$-flatness of $\tilde{g}_{t}$ at $\pi^{-1}(D)$ implies that $\tilde{k}(p, t) f_{0}(\pi(p))=\tilde{g}(p, t)$, where $\tilde{k}: \tilde{X} \times$ $[0,1] \rightarrow \mathbf{R}$ is an analytic function vanishing on $\pi^{-1}(D) \times[0,1]$.

We now proceed towards the main result of this section.

Let $X$ be a real analytic manifold. Denote by $D^{r}(X)$ the group of $C^{r}$ diffeomorphisms of $X, r=0,1,2, \ldots, \omega$, where $\omega$ stands for analytic. The ring of analytic functions from $X$ to $\mathbf{R}$ will be denoted by $\mathscr{O}(X)$ and the ring of analytic function-germs $(X, x) \rightarrow \mathbf{R}$ at $x$ in $X$ by $\mathscr{O}_{X}(X)$.

Let $\xi=(E, \rho, X)$ be an analytic vector bundle over $X$. Denote by $\Gamma^{r}(\xi)$ the space of $C^{r}$ sections of $\xi$ and by $E_{x}$ the fibre of $\xi$ at $x$. If $s$ is a section of $\xi$, then $s^{-1}(0)$ will denote the set of zeros of $s$. If $s$ is analytic, then we define the ideal $I(s)$ of $\mathscr{O}(X)$ as follows: Given $x$ in $X$, let $\left(s_{1}, \ldots, s_{k}\right)$ be a system of germs at $x$ of analytic sections of $\xi$ such that $\left(s_{1}(x), \ldots, s_{k}(x)\right)$ is a basis for $E_{x}$. Then the germ $s_{x}$ of $s$ at $x$ can be written as $s_{x}=f_{1} s_{1}+\cdots+f_{k} s_{k}$ for some $f_{i}$ in $\mathscr{O}_{x}(X)$. We define $I(s)$ to be the unique ideal of $\mathscr{O}(X)$ satisfying

$$
I(s) \mathscr{O}_{x}(X)=\sum_{i=1}^{k} f_{i} \mathscr{O}_{x}(X)
$$

for all $x$ in $X$. Observe that the set of zeros of $I(s)$ is equal to $s^{-1}(0)$. Analogously, if $\Phi$ is an analytic section over $X \times[0,1]$ of $\xi$, i.e., $\Phi$ is an analytic map from $X \dot{x}[0,1]$ to $E$ such that $\Phi_{t}=\Phi(\cdot, t)$ is a section of $\xi$ for all $t$ in $[0,1]$, then the germ $\Phi_{(x, t)}$ of $\Phi$ at $(x, t)$ can be written as $\Phi_{(x, t)}=F_{1} s_{1}+\cdots+F_{k} s_{k}$ for some $F_{i}$ in $\mathscr{O}_{(x, t)}(X \times[0,1])$. We define $I(\Phi)$ to be the unique ideal of $\mathscr{O}(X \times[0,1])$ satisfying

$$
I(\Phi) \mathscr{O}_{(x, t)}(X \times[0,1])=\sum_{i=1}^{k} F_{i} \mathscr{O}_{(x, t)}(X \times[0,1])
$$

for all $(x, t)$ in $X \times[0,1]$.

Let $\pi: \widetilde{X} \rightarrow X$ be an analytic map between real analytic manifolds (in our applications, $X$ will be an affine real algebraic variety and $\pi$ will be an algebraic multiblowup of $X$ along a subvariety consisting of finitely many points). Let $\xi_{i}=\left(E_{i}, \rho_{i}, X\right), i=1, \ldots, d$, be analytic vector bundles over $X, \operatorname{rank} \xi_{i}=c(i)$, and let $\xi=(E, \rho, X)=$ $\xi_{1} \oplus \cdots \oplus \xi_{d}$. 
The set

$$
\begin{array}{r}
G^{r}(\xi)=\left\{(\gamma, \sigma) \in D^{r}(E) \times D^{r}(X) \mid \rho \circ \gamma=\sigma \circ \rho,\right. \\
\gamma\left(E_{i}\right) \subset E_{i} \text { for } i=1, \ldots, \text { and } \gamma \mid E_{x}: E_{x} \rightarrow E_{\sigma(x)} \\
\text { is a linear isomorphism for } x \in X\}
\end{array}
$$

is a subgroup of $D^{r}(E) \times D^{r}(X)$. The action of $G^{r}(\xi)$ on $\Gamma^{r}(\xi)$ is defined by

$$
(\gamma, \sigma) \cdot s=\gamma \circ s \circ \sigma^{-1}
$$

for $(\gamma, \sigma)$ in $G^{r}(\xi)$ and $s$ in $\Gamma^{r}(\xi)$. Let $G^{r}(\xi, \pi)$ be the subgroup of $G^{r}(\xi)$ consisting of all elements $(\gamma, \sigma)$ in $G^{r}(\xi)$ such that there is an analytic diffeomorphism $\tilde{\sigma}: \widetilde{X} \rightarrow \tilde{X}$ satisfying $\sigma \circ \pi=\pi \circ \tilde{\sigma}$. We say that two sections in $\Gamma^{r}(\xi)$ are $\pi$-analytically $C^{r}$ equivalent if they are in the same $G^{r}(\xi, \pi)$-orbit. Note that if $s=s_{1} \oplus \cdots \oplus s_{d}$ and $u=u_{1} \oplus \cdots \oplus u_{d}$ are $C^{r}$ sections of $\xi$ and $u=(\gamma, \sigma) \cdot s$, then $\sigma\left(s_{i}^{-1}(0)\right)=u_{i}^{-1}(0)$ for $i=1, \ldots, d$.

Let $c=(c(1), \ldots, c(d))$ and $|c|=c(1)+\cdots+c(d)$. Denote by $G(c)$ the subgroup of $\mathrm{Gl}(|c|, \mathbf{R})$ consisting of all matrices of the form

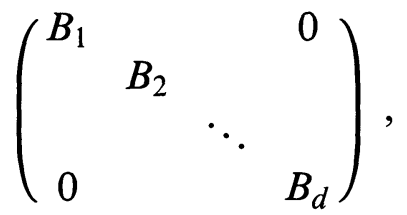

where $B_{i}$ is in $\operatorname{Gl}(c(i), \mathbf{R}), i=1, \ldots, d$. let $\left\{A_{j}\right\}, j=1, \ldots, q$, $q=c(1)^{2}+\cdots+c(d)^{2}$, be a basis for the tangent space $T_{I} G(c)$ of $G(c)$ at the identity $|c| \times|c|$ matrix $I$ (we consider $T_{I} G(c)$ embedded in the space of all $|c| \times|c|$ matrices). Given

$$
f=\left(f_{11}, \ldots, f_{1 c(1)}, \ldots, f_{d 1}, \ldots, f_{d c(d)}\right)
$$

in $\mathscr{O}(X)^{|c|}$, we define $J(f)$ to be the ideal of $\mathscr{O}(X)$ generated by all $|c| \times|c|$ minors of the $|c| \times(m+q)$ matrix $L(f)$ with $A_{1} \cdot f, \ldots$, $A_{q} \cdot f, Y_{1} f, \ldots, Y_{m} f$ as columns,

$$
L(f)=\left(A_{1} \cdot f, \ldots, A_{q} \cdot f, Y_{1} f, \ldots, Y_{m} f\right),
$$

where $\left\{Y_{l}\right\}_{l=1, \ldots, m}$ are analytic vector fields on $X$ generating the $\mathscr{O}(X)$-module $\Gamma^{\omega}(T X)$. Similarly, if

$$
F=\left(F_{11}, \ldots, F_{1 c(1)}, \ldots, F_{d 1}, \ldots, F_{d c(d)}\right)
$$

is in $\mathscr{O}(X \times[0,1])^{|c|}$ and the $Y_{l}$ are considered, in the obvious way, as elements of $\Gamma^{\omega}(T(X \times[0,1]))$, then we let $J(F)$ denote the ideal of $\mathscr{O}(X \times[0,1])$ generated by all $|c| \times|c|$ minors of the matrix

$$
L(F)=\left(A_{1} \cdot F, \ldots, A_{q} \cdot F, Y_{1} F, \ldots, Y_{m} F\right) .
$$


Given an analytic section $s=s_{1} \oplus \cdots \oplus s_{d}$ of $\xi$, let $J(s)$ denote the ideal of $\mathscr{O}(X)$ generated by all $J(f)$ with $f_{i j}$ in $I\left(s_{i}\right)$ for $i=$ $1, \ldots, d, j=1, \ldots, c(i)$. If $\Phi=\Phi_{1} \oplus \cdots \oplus \Phi_{d}$ is an analytic section over $X \times[0,1]$ of $\xi$, then $J(\Phi)$ will denote the ideal of $\mathscr{O}(X \times[0,1])$ generated by all $J(F)$ with $F_{i j}$ in $I\left(\Phi_{i}\right)$ for $i=1, \ldots, d, j=$ $1, \ldots, c(i)$.

The geometric significance of the ideal $J(s)$ is explained below $(J(\Phi)$ is introduced for technical reasons).

LEMMA 2.4. Let $s=s_{1} \oplus \cdots \oplus s_{d}$ be an analytic section of $\xi$ and let $Z_{i}=s_{i}^{-1}(0), i=1, \ldots, d$. Assume that $I\left(s_{i}\right)$ is equal to the ideal of all functions in $\mathscr{O}(X)$ vanishing on $Z_{i}$ and codim $Z_{i}=c(i)$. Then the following conditions are equivalent:

(i) Each $Z_{i}$ is an analytic submanifold of $X$ and the family $\left\{Z_{1}, \ldots, Z_{d}\right\}$ is in general position.

(ii) The set of zeros of $J(s)$ is empty.

Proof. An elementary argument of linear algebra.

We are now ready to prove the main result of this section.

Proposition 2.5. Let $X$ be a compact affine nonsingular real algebraic variety. Let $\xi_{i}=\left(E_{i}, \rho_{i}, X\right), i=1, \ldots, d$, be analytic vector bundles over $X$ and let $s=s_{1} \oplus \cdots \oplus s_{d}$ be an analytic section of $\xi=\xi_{1} \oplus \cdots \oplus \xi_{d}$. Let $\delta$ be an element of $J(s)$ such that the set $\delta^{-1}(0)=D$ is finite and

$$
\delta(x) \geq a \operatorname{dist}(x, D)^{m} \text { for all } x \text { in } X,
$$

where $a>0$ and $m$ is a positive integer. Let $\pi: \widetilde{X} \rightarrow X$ be an algebraic $k$-fold multiblowup of $X$ along $D$ such that $\delta \circ \pi$ is locally a normal crossing. Then there exists a neighborhood $\mathscr{V}$ of 0 in $\Gamma^{\omega}(\xi)$ (in the $C^{\infty}$ topology) such that for every positive integer $r$ and every analytic section $u$ of $\xi$, if $u-s$ belongs to $\mathscr{V}$ and is l-flat at $D$, $l=\max \{k+h(\delta, \pi),(r+1) m\}$, then $u$ is $\pi$-analytically $C^{r}$ equivalent to $s$.

Proof. First we shall replace the vector bundles $\xi_{i}$ by trivial vector bundles over a "larger" manifold $\Omega$.

Let $\langle,\rangle_{i}$ be an analytic Riemannian metric on $\xi_{i}$ and let $c(i)=$ rank $\xi_{i}$. The set

$$
\Omega=\left\{(\varepsilon, x) \in E_{1}^{c(1)} \times \cdots \times E_{d}^{c(d)} \times X \mid \varepsilon=\left(\varepsilon_{1}, \ldots, \varepsilon_{d}\right),\right.
$$

$\varepsilon_{i}$ is an orthonormal basis for $\left.E_{i x}, i=1, \ldots, d\right\}$ 
is a compact analytic submanifold of $E_{1}^{c(1)} \times \cdots \times E_{d}^{c(d)} \times X$ and the map

$$
\Lambda: \Omega \rightarrow X, \quad \Lambda(\varepsilon, x)=x
$$

is an analytic, locally trivial fibration. The fibres of $\Lambda$ are diffeomorphic to the product $V\left(\mathbf{R}^{c(1)}\right) \times \cdots \times V\left(\mathbf{R}^{c(d)}\right)$, where $V\left(\mathbf{R}^{k}\right)$ stands for the Stiefel manifold of orthonormal bases for $\mathbf{R}^{k}$.

Let $O(c)=O(c(1)) \times \cdots \times O(c(d))$, where $O(k)$ denotes the group of orthogonal $k \times k$ matrices. We shall consider $O(c)$ as a subgroup of $G(c)$. Define the action of $O(c)$ on $\Omega$ by the following formula:

$$
\alpha \cdot(\varepsilon, x)=(\alpha \cdot \varepsilon, x),
$$

where $\alpha$ is in $O(c),(\varepsilon, x)$ is in $\Omega$ and $\varepsilon$ is considered as a $|c| \times 1$ matrix.

For each $i=1, \ldots, d$, the pull-back vector bundle $\Lambda^{*} \xi_{i}=$ $\left(\Lambda^{*} E_{i}, \Lambda^{*} \rho_{i}, \Omega\right)$ is trivial. Indeed, by definition

$$
\Lambda^{*} E_{i}=\left\{(\omega, e) \in \Omega \times E_{i} \mid \Lambda(\omega)=\rho_{i}(e)\right\}
$$

and the analytic sections

$$
s_{i j}: \Omega \rightarrow \Lambda^{*} E_{i}
$$

of $\Lambda^{*} \xi_{i}$ given by

$$
s_{i j}(\varepsilon, x)=\left((\varepsilon, x), \varepsilon_{i j}\right),
$$

where $\varepsilon=\left(\varepsilon_{1}, \ldots, \varepsilon_{d}\right), \varepsilon_{i}=\left(\varepsilon_{i 1}, \ldots, \varepsilon_{i c(i)}\right)$, are linearly independent at every point $(\varepsilon, x)$ in $\Omega$ for $j=1, \ldots, c(i)$.

Given a section $v=v_{1} \oplus \cdots \oplus v_{d}$ of $\xi$, let

$$
f_{v}=\left(f_{v 11}, \ldots, f_{v 1 c(1)}, \ldots, f_{v d 1}, \ldots, f_{v d c(d)}\right)
$$

be the element of $\mathscr{O}(\Omega)^{|c|}$ determined by the equations

$$
\Lambda^{*} v_{i}=\sum_{j=1}^{c(i)} f_{v i j} s_{i j}, \quad i=1, \ldots, d,
$$

where $\Lambda^{*} v_{i}: \Omega \rightarrow \Lambda^{*} E_{i}$ is the pull-back of $v_{i}$, that is,

$$
\left(\Lambda^{*} v_{i}\right)(\omega)=\left(\omega, v_{i}(\Lambda(\omega))\right)
$$

for $\omega$ in $\Omega$.

One sees immediately that $f_{v}$ (considered as a map from $\Omega$ to $\left.\mathbf{R}^{|c|}\right)$ is $O(c)$-equivariant, where the action of $O(c)$ on $\mathbf{R}^{|c|}$ is the usual matrix multiplication. 
Now, let $u$ be an analytic section of $\xi$ such that $u-s$ is $l$-flat at $D$. Set $\Phi(x, t)=(1-t) s(x)+t u(x)$ for $(x, t)$ in $X \times[0,1]$ and pick an element $\Delta$ in $J(\Phi)$ such that $\Delta_{0}=\delta$ and $\Delta_{t}-\delta$ is $(l-1)$-flat at $D$ for $t$ in $[0,1]$. If $u$ is sufficiently close to $s$ (where the closeness does not depend on $r$ ), then $\Delta_{t}^{-1}(0)=D$ for $t$ in $[0,1]$ and

$$
\Delta(x, t) \geq b \operatorname{dist}(x, D)^{m} \text { for all }(x, t) \text { in } X \times[0,1],
$$

where $b>0$.

Define $F: \Omega \times[0,1] \rightarrow \mathbf{R}^{|c|}$ and $\Sigma: \Omega \times[0,1] \rightarrow \mathbf{R}$ by

$$
\begin{aligned}
& F(\omega, t)=f_{\Phi_{t}}(\omega), \\
& \Sigma(\omega, t)=\Delta(\Lambda(\omega), t)
\end{aligned}
$$

for $(\omega, t)$ in $\Omega \times[0,1]$. Computing in a local coordinate system, one easily sees that for each point $(\omega, t)$ in $\Omega \times[0,1]$ the germ of $\Sigma$ at $(\omega, t)$ is in $J(F) \mathscr{O}_{(\omega, t)}(\Omega \times[0,1])$. It follows that $\Sigma$ is in $J(F)$ and, by Cramer's rule, for every element $H$ of $\mathscr{O}(\Omega \times[0,1])^{|c|}$, the product $\Sigma H$ is a linear combination with coefficients in $\mathscr{O}(\Omega \times[0,1])$ of the columns of the matrix $L(F)$. Hence

$$
\text { (2.5.2) }-\Sigma(\omega, t) \frac{\partial F}{\partial t}(\omega, t)=d_{\omega} F_{t}\left(\nu^{\prime}(\omega, t)\right)+\kappa^{\prime}(\omega, t) \cdot F(\omega, t),
$$

where $\nu^{\prime}$ is a time-dependent analytic vector field on $\Omega$ and $\kappa^{\prime}: \Omega \times$ $[0,1] \rightarrow T_{I} G(c)$ is an analytic map. Moreover, since $\partial F / \partial t$ is $l$-flat at $\Lambda^{-1}(D)$ for all $t$ in $[0,1]$, we may assume that

$$
\nu_{t}^{\prime} \text { and } \kappa_{t}^{\prime} \text { are } l \text {-flat at } \Lambda^{-1}(D) \text { for } t \text { in }[0,1] \text {. }
$$

Using the fact that $F_{t}$ is $O(c)$-equivariant and eventually replacing in (2.5.2) $\nu_{t}^{\prime}=\nu^{\prime}(\cdot, t)$ by its $O(c)$-average and $\kappa^{\prime}(\omega, t)$ by

$$
\int_{O(c)} \alpha^{-1} \cdot \kappa^{\prime}(\alpha \cdot \omega, t) \cdot \alpha d \mu
$$

where $\alpha$ is in $O(c)$ and $\mu$ is the Haar measure on $O(c)$ with $\int d \mu=$ 1 , we may assume that $\nu_{t}^{\prime}$ is an $O(c)$-equivariant vector field and $\kappa^{\prime}$ satisfies

$$
\kappa^{\prime}(\alpha \cdot \omega, t)=\alpha \cdot \kappa^{\prime}(\omega, t) \cdot \alpha^{-1} .
$$

Since $\Lambda$ is a fibration, one easily sees that

$$
\Sigma(\omega, t) \geq d \operatorname{dist}\left(\omega, \Lambda^{-1}(D)\right)^{m} \text { for }(\omega, t) \text { in } \Omega \times[0,1]
$$


where $d>0$ (cf. (2.5.1)). It follows from this inequality and (2.5.3) that the time-dependent vector field

$$
\nu^{*}(\omega, t)= \begin{cases}\nu^{\prime}(\omega, t) / \Sigma(\omega, t) & \text { for }(\omega, t) \in(\Omega \times[0,1]) \backslash \Sigma^{-1}(0), \\ 0 & \text { for }(x, t) \in \Sigma^{-1}(0)\end{cases}
$$

is of class $C^{r}$ and

$$
\kappa^{*}(\omega, t)= \begin{cases}\kappa^{\prime}(\omega, t) / \Sigma(\omega, t) & \text { for }(\omega, t) \in(\Omega \times[0,1]) \backslash \Sigma^{-1}(0), \\ 0 & \text { for }(\omega, t) \in \Sigma^{-1}(0)\end{cases}
$$

is also of class $C^{r}$. From (2.5.2) we obtain

$$
-\frac{\partial F}{\partial t}(\omega, t)=d_{\omega} F_{t}\left(\nu^{*}(\omega, t)\right)+\kappa^{*}(\omega, t) \cdot F(\omega, t) .
$$

Let $\left\{\sigma_{t}^{*}=\sigma^{*}(\cdot, t)\right\}, t \in[0,1]$, be the $C^{r}$ diffeotopy of $\Omega$ determined by $\nu^{*}$, that is,

$$
\left\{\begin{array}{l}
\frac{\partial \sigma^{*}}{\partial t}(\omega, t)=\nu^{*}\left(\sigma^{*}(\omega, t), t\right) \\
\sigma^{*}(\omega, 0)=\omega
\end{array}\right.
$$

and let $R^{*}: \Omega \times[0,1] \rightarrow \mathrm{GL}(|c|, \mathbf{R})$ be the resolvent of $-\kappa^{*}\left(\sigma^{*}(\omega, t), t\right)$, that is, $R^{*}$ is a unique $C^{r}$ map satisfying

$$
\left\{\begin{array}{l}
\frac{\partial R^{*}}{\partial t}(\omega, t)=-\kappa^{*}\left(\sigma^{*}(\omega, t), t\right) \cdot R^{*}(\omega, t), \\
R^{*}(\omega, 0)=I \text { (the identity matrix). }
\end{array}\right.
$$

Since $\kappa^{*}\left(\sigma^{*}(\omega, t), t\right)$ is in $T_{I} G(c)$, we obtain that $R^{*}(\omega, t)$ is in $G(c)$ for all $(\omega, t)$ in $\Omega \times[0,1]$.

Using (2.5.5), (2.5.6) and (2.5.7), we obtain immediately

$$
\left(F_{t} \circ \sigma_{t}^{*}\right)(\omega)=R^{*}(\omega, t) \cdot F_{0}(\omega)
$$

for $(\omega, t)$ in $\Omega \times[0,1]$.

Clearly, $\left\{\sigma_{t}^{*}\right\}$ is $O(c)$-equivariant. Hence there exists a unique $C^{r}$ diffeotopy $\left\{\sigma_{t}\right\}$ of $X$ satisfying $\sigma_{t} \circ \Lambda=\Lambda \circ \sigma_{t}^{*}$.

Let

$$
\begin{aligned}
& \omega=(\varepsilon, x) \in \Omega, \quad \varepsilon=\left(\varepsilon_{1}, \ldots, \varepsilon_{d}\right), \quad \varepsilon_{i}=\left(\varepsilon_{i 1}, \ldots, \varepsilon_{i c(i)}\right), \\
& \sigma_{t}^{*}(\omega)=\left(\varepsilon^{t}, \sigma_{t}(x)\right) \in \Omega, \quad \varepsilon^{t}=\left(\varepsilon_{1}^{t}, \ldots, \varepsilon_{d}^{t}\right), \quad \varepsilon_{i}^{t}=\left(\varepsilon_{i 1}^{t}, \ldots, \varepsilon_{i c(i)}^{t}\right) .
\end{aligned}
$$

We define $\gamma_{t}: E \rightarrow E$ as follows: Given $e$ in $E=E_{1} \oplus \cdots \oplus E_{d}$, we can write

$$
e=\sum_{i=1}^{d} \sum_{j(i)=1}^{c(i)} \lambda_{i j(i)} \varepsilon_{i j(i)}
$$


where $\lambda_{i j(i)} \in \mathbf{R}$ are uniquely determined. Let

$$
\lambda=\left(\lambda_{11}, \ldots, \lambda_{1 c(1)}, \ldots, \lambda_{d 1}, \ldots, \lambda_{d c(d)}\right)
$$

and

$$
R^{*}(\omega, t)(\lambda)=\lambda^{t}
$$

where

$$
\lambda^{t}=\left(\lambda_{11}^{t}, \ldots, \lambda_{1 c(1)}^{t}, \ldots, \lambda_{d 1}^{t}, \ldots, \lambda_{d c(d)}^{t}\right) .
$$

Define $\gamma_{t}: E \rightarrow E$ by

$$
\gamma_{t}(e)=\sum_{i=1}^{d} \sum_{j(i)=1}^{c(i)} \lambda_{i j(i)}^{t} \varepsilon_{i j(i)}^{t} .
$$

This construction makes sense (i.e., $\gamma_{t}$ is well-defined and of class $C^{r}$ ) since $\sigma_{t}^{*}$ is $O(c)$-equivariant and, by (2.5.4), $R^{*}$ satisfies

$$
R^{*}(\alpha \cdot \omega, t)=\alpha \cdot R^{*}(\omega, t) \cdot \alpha^{-1}
$$

for all $(\omega, t)$ in $\Omega \times[0,1]$ and $\alpha$ in $O(c)$. Observe that $\left(\gamma_{t}, \sigma_{t}\right)$ is in $G^{r}(\xi)$. Using $(2.5 .8)$, one obtains

$$
\Phi_{t}=\left(\gamma_{t}, \sigma_{t}\right) \cdot \Phi_{0}
$$

Since $\Phi_{0}=s$ and $\Phi_{1}=u$, we have, in particular,

$$
u=\left(\gamma_{1}, \sigma_{1}\right) \cdot s .
$$

Thus to complete the proof it suffices to show that $\left(\gamma_{t}, \sigma_{t}\right)$ belongs to $G^{r}(\xi, \pi)$.

Since $\nu_{t}^{*}$ is $O(c)$-equivariant, it follows that there exists a timedependent $C^{r}$ vector field $\nu$ on $X$ satisfying

$$
\nu(\Lambda(\omega), t)=d_{\omega} \Lambda\left(\nu^{*}(\omega, t)\right)
$$

for all $(\omega, t)$ in $\Omega \times[0,1]$.

We claim that the time-dependent vector field $\tilde{\nu}$ on $\widetilde{X}$ defined by $\tilde{\nu}(p, t)= \begin{cases}\left(d_{p} \pi\right)^{-1}(\nu(\pi(p), t)) & \text { for }(p, t) \in\left(\tilde{X} \backslash \pi^{-1}(D)\right) \times[0,1], \\ 0 & \text { for }(p, t) \in \pi^{-1}(D) \times[0,1]\end{cases}$

is analytic. Indeed, since $\nu_{t}^{\prime}$ is $O(c)$-equivariant, there exists a timedependent analytic vector field $\nu^{1}$ on $X$ satisfying

$$
\nu^{1}(\Lambda(\omega), t)=d_{\omega} \Lambda\left(\nu^{\prime}(\omega, t)\right)
$$


for all $(\omega, t)$ in $\Omega \times[0,1]$. Clearly, $\nu_{t}^{1}$ is $l$-flat at $D$ for all $t$ in $[0,1]$ and by Lemma 2.1 , the time-dependent vector field $\tilde{\nu}^{1}$ on $\widetilde{X}$ defined by

$\tilde{\nu}^{1}(p, t)= \begin{cases}\left(d_{p} \pi\right)^{-1}\left(\nu^{1}(\pi(p), t)\right) & \text { for }(p, t) \in\left(\widetilde{X} \backslash \pi^{-1}(D)\right) \times[0,1], \\ 0 & \text { for }(p, t) \in \pi^{-1}(D) \times[0,1]\end{cases}$

is analytic and $h(\delta, \pi)$-flat at $D$. Since

$$
\tilde{\nu}^{1}(p, t)=\Delta(\pi(p), t) \tilde{\nu}(p, t)
$$

for $(p, t)$ in $\tilde{X} \times[0,1],(2.5 .1)$ and Lemma 2.3 imply that $\tilde{\nu}$ is analytic. Thus the claim is proved.

Note that $\left\{\sigma_{t}\right\}$ is the diffeotopy of $X$ determined by $\nu$. If $\left\{\tilde{\sigma}_{t}\right\}$ is the analytic diffeotopy of $\tilde{X}$ determined by $\tilde{\nu}$, then $\pi \circ \tilde{\sigma}_{t}=\sigma_{t} \circ \pi$. Hence $\left(\gamma_{t}, \sigma_{t}\right)$ belongs to $G^{r}(\xi, \pi)$ and the proof of Proposition 2.5 is finished.

3. Proof of Theorem 1.1. Let $X$ be an affine real algebraic variety of dimension $n$. An algebraic vector bundle $\xi$ over $X$ is said to be strongly algebraic if there exists an algebraic vector bundle $\eta$ over $X$ such that $\xi \oplus \eta$ is algebraically trivial, i.e., algebraically isomorphic to a product vector bundle $X \times \mathbf{R}^{m}$ (cf. [7], Theorem 12.1.7 or [2]).

Let $V$ be a coherent analytic subset of $X$ of pure dimension $n-1$ at each point. One constructs, in the standard way, an analytic real line bundle $\xi$ over $X$ and an analytic section $s$ of $\xi$ such that $I(s)$ is equal to the ideal of all functions in $\mathscr{O}(X)$ vanishing on $V$ (in particular, $\left.s^{-1}(0)=V\right)$. Then the first Stiefel-Whitney class $w_{1}(\xi)$ of $\xi$ is Poincare dual to the homology class [ $V$ ] in $H_{n-1}(X, \mathbf{Z} / 2)$ represented by $V$. Recall that $\xi$ is topologically (and hence also analytically) isomorphic to a strongly algebraic line bundle over $X$ if and only if [ $V$ ] belongs to $H_{n-1}^{\text {alg }}(X, \mathrm{Z} / 2)$ [7], Theorem 12.4 .8 or [3].

Proof of Theorem 1.1. Let $\xi_{i}$ be an analytic line bundle over $X$ and let $s_{i}$ be an analytic section of $\xi_{i}$ such that $I\left(s_{i}\right)$ is equal to the ideal of $\mathscr{O}(X)$ of all functions vanishing on $V_{i}$. Since $\left[V_{i}\right]$ is in $H_{n-1}^{\text {alg }}(X, \mathbf{Z} / 2)$, we may assume that $\xi_{i}$ is a strongly algebraic line bundle (cf. the remark above). Set $\xi=\xi_{1} \oplus \cdots \oplus \xi_{d}$ and $s=s_{1} \oplus \cdots \oplus s_{d}$. By Lemma 2.4, the set of zeros of the ideal $J(s)$ is contained in $D$. Thus one can find an element $\delta$ in $J(s)$ with $\delta^{-1}(0)=D$ and $\delta \geq 0$. By the Lojasiewicz inequality [16],

$$
\delta(x) \geq a \operatorname{dist}(x, D)^{m} \text { for all } x \text { in } X,
$$


where $a>0$ and $m$ is a positive integer. It follows from Lemma 2.2 that there exists an algebraic, say, $k$-fold multiblowup $\pi: \widetilde{X} \rightarrow X$ such that $\delta \circ \pi$ is locally a normal crossing.

Let $r$ be a positive integer and let

$$
l=\max \{k+h(\delta, \pi),(r+1) m\} .
$$

Since $\xi$ is a strongly algebraic vector bundle over $X$, one can find an algebraic section $u=u_{1} \oplus \cdots \oplus u_{d}$ of $\xi$ such that $u$ is close to $s$ in the $C^{\infty}$ topology and $u-s$ is l-flat at $D$ (cf. [4], Corollary 1). By Proposition 2.5, there exists an element $(\gamma, \sigma)$ in $G^{r}(\xi, \pi)$ satisfying $u=(\gamma, \sigma) \cdot s$. Hence, in particular, $\sigma\left(s_{i}^{-1}(0)\right)=u_{i}^{-1}(0)$ for $i=1, \ldots, d$ and $\pi \circ \tilde{\sigma}=\sigma \circ \pi$ for some analytic diffeomorphism $\tilde{\sigma}$ of $\widetilde{X}$. Since $u_{i}^{-1}(0)$ is an algebraic subvariety of $X$ and $V_{i}=s_{i}^{-1}(0)$, the proof of Theorem 1.1 is complete.

Acknowledgment. I wish to thank the referee for his or her comments on the first version of this paper.

\section{REFERENCES}

[1] S. Akbulut and H. King, The topology of real algebraic sets, Enseign. Math., 29 (1983), 221-261.

[2] R. Benedetti and A. Tognoli, On real algebraic vector bundles, Bull. Sci. Math., 104 (1980), 89-112.

[3] _ Remarks and counterexamples in the theory of real algebraic vector bundles and cycles, In: Géométrie Algébrique Réelle et Formes Quadratiques. Lecture Notes in Math. vol. 959, pp. 198-211. Berlin, Heidelberg, New York: Springer 1982.

[4] J. Bochnak, W. Kucharz and M. Shiota, On equivalence of ideals of real global analytic functions and the 17 th Hilbert problem, Invent. Math., 63 (1981), 403421.

[5] _- On algebraicity of global real analytic sets and functions, Invent. Math., 70 (1982), 115-156.

[6] J. Bochnak and W. Kucharz, Local algebraicity of analytic sets, J. Reine Angew. Math., 352 (1984), 1-14.

[7] J. Bochnak, M. Coste and M.-F. Roy. Géométrie Algébrique Réele, Ergebnisse der Math. vol. 12, Berlin, Heidelberg, New York: Springer 1987.

[8] A. Borel and A. Haefliger, La classe d'homologie fondamentale d'un espace analytique, Bull. Soc. Math. France, 89 (1961), 461-513.

[9] M. Buchner and W. Kucharz, Almost analytic local algebraicity of analytic sets and functions, Math. Z., 196 (1987), 65-74.

[10] H. Hironaka, Resolution of singularities of an algebraic variety over a field of characteristic zero, Ann. of Math., 79 (1964), 205-326.

[11] T. C. Kuo and J. N. Ward, A theorem on almost analytic equisingularity, J. Math. Soc. Japan, 33 (1981), 471-488. 
[12] T. C. Kuo, The modified analytic trivialization of singularities, J. Math. Soc. Japan, 32 (1980), 605-614.

[13] _ Une classification des singularités isolées C. R. Acad. Sci. Paris, 288 (1979), 809-812.

[14] _ - On classification of real singularities, Invent. Math., 82 (1985), 257-262.

[15] J.-P. Serre, Faisceaux algébriques cohérents, Ann. of Math., 61 (1955), 197-278.

[16] J.-Cl. Tougeron, Idéaux de Fonctions Différentiables, Ergebnisse der Math., vol. 71, Berlin, Heidelberg, New York: Springer 1972.

Received February 3, 1987 and in revised form July 27, 1989.

University of New MeXico

ALbUQUeRQUe, NM 87131

Current address: Department of Mathematics

University of Hawaii at Manoa

2565 The Mall

Honolulu, HI 96822 


\section{PACIFIC JOURNAL OF MATHEMATICS EDITORS}

\author{
V. S. VARADARAJAN \\ (Managing Editor) \\ University of California \\ Los Angeles, CA 90024-1555-05 \\ Herbert Clemens \\ University of Utah \\ Salt Lake City, UT 84112 \\ ThOMAs ENRIGHT \\ University of California, San Diego \\ La Jolla, CA 92093
}

R. FINN

Stanford University

Stanford, CA 94305

HeRmanN FlaschKa

University of Arizona

Tucson, AZ 85721

VAUGHAN F. R. JoNES

University of California

Berkeley, CA 94720

SteVen KerCKhofF

Stanford University

Stanford, CA 94305
ROBION KIRBY

University of California

Berkeley, CA 94720

C. C. Moore

University of California

Berkeley, CA 94720

HAROLD STARK

University of California, San Diego

La Jolla, CA 92093

\section{ASSOCIATE EDITORS}
R. ARENS
E. F. BECKENBACH
B. H. NeumanN
F. Wolf
K. YoshidA
(1906-1982)
(1904-1989)

\section{SUPPORTING INSTITUTIONS}

UNIVERSITY OF ARIZONA
UNIVERSITY OF BRITISH COLUMBIA
CALIFORNIA INSTITUTE OF TECHNOLOGY
UNIVERSITY OF CALIFORNIA
MONTANA STATE UNIVERSITY
UNIVERSITY OF NEVADA, RENO
NEW MEXICO STATE UNIVERSITY
OREGON STATE UNIVERSITY
UNIVERSITY OF ARIZONA
UNIVERSITY OF BRITISH COLUMBIA
UNIVERSITY OF CALIFORNIA
MONTANA STATE UNIVERSITY
NEW MEXICO STATE UNIVERSITY

\author{
UNIVERSITY OF OREGON \\ UNIVERSITY OF SOUTHERN CALIFORNIA \\ STANFORD UNIVERSITY \\ UNIVERSITY OF HAWAII \\ UNIVERSITY OF TOKYO \\ UNIVERSITY OF UTAH \\ WASHINGTON STATE UNIVERSITY \\ UNIVERSITY OF WASHINGTON
}

The Supporting Institutions listed above contribute to the cost of publication of this Journal, but they are not owners or publishers and have no responsibility for its content or policies.

Mathematical papers intended for publication in the Pacific Journal of Mathematics should be in typed form or offset-reproduced (not dittoed), double spaced with large margins. Please do not use built up fractions in the text of the manuscript. However, you may use them in the displayed equations. Underline Greek letters in red, German in green, and script in blue. The first paragraph must be capable of being used separately as a synopsis of the entire paper. In particular it should contain no bibliographic references. Please propose a heading for the odd numbered pages of less than 35 characters. Manuscripts, in triplicate, may be sent to any one of the editors. Please classify according to the 1980 Mathematics Subject Classification (1985 Revision) scheme which can be found in the December index volumes of Mathematical Reviews. Supply name and address of author to whom proofs should be sent. All other communications should be addressed to the managing editor, or Elaine Barth, University of California, Los Angeles, California 90024-1555-05.

There are page-charges associated with articles appearing in the Pacific Journal of Mathematics. These charges are expected to be paid by the author's University, Government Agency or Company. If the author or authors do not have access to such Institutional support these charges are waived. Single authors will receive 50 free reprints; joint authors will receive a total of 100 free reprints. Additional copies may be obtained at cost in multiples of 50 .

The Pacific Journal of Mathematics is issued monthly as of January 1966. Regular subscription rate: $\$ 190.00$ a year (6 Vols., 12 issues). Special rate: $\$ 95.00$ a year to individual members of supporting institutions.

Subscriptions, orders for numbers issued in the last three calendar years, and changes of address should be sent to Pacific Journal of Mathematics, P.O. Box 969, Carmel Valley, CA 93924, U.S.A. Old back numbers obtainable from Kraus Periodicals Co., Route 100, Millwood, NY 10546.

The Pacific Journal of Mathematics at P.O. Box 969, Carmel Valley, CA 93924 (ISSN 0030-8730) is published monthly. Second-class postage paid at Carmel Valley, California 93924, and additional mailing offices. Postmaster: send address changes to Pacific Journal of Mathematics, P.O. Box 969, Carmel Valley, CA 93924.

\section{PUBLISHED BY PACIFIC JOURNAL OF MATHEMATICS, A NON-PROFIT CORPORATION}




\section{Pacific Journal of Mathematics}

\section{Vol. 143, No. $2 \quad$ April, 1990}

Gustavo Corach, Horacio Porta and Lázaro Recht, Differential geometry of systems of projections in Banach algebras ................. 209

Peter Fleischmann and Jens Carsten Jantzen, Simple periodic modules of

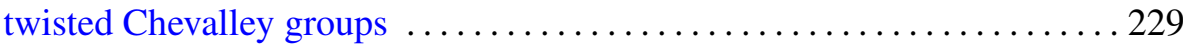

Niels Gronbaek, Amenability of discrete convolution algebras, the commutative case ...................................243

Nguyên H. V. Hung, The mod 2 equivariant cohomology algebras of

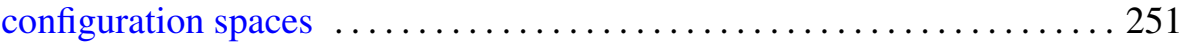

Wojciech Kucharz, Global almost analytic algebraicity of analytic sets . . . 287 John Merrill, A class of consistent anti-Martin's axioms .............. 301

Takafumi Murai, The power 3/2 appearing in the estimate of analytic

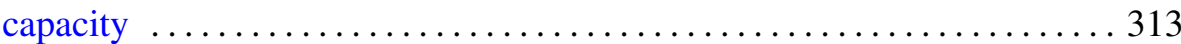

L. Panaitopol and Doru Stefanescu, On the generalized difference

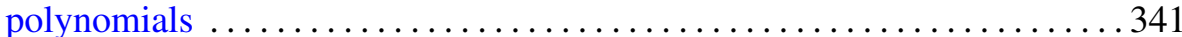

Katsuhiro Shiohama, Takashi Shioya and Minoru Tanaka, Mass of rays

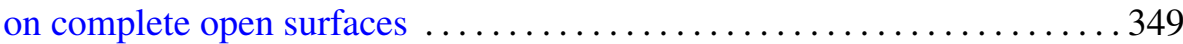

Gerhard Ströhmer, About compressible viscous fluid flow in a bounded

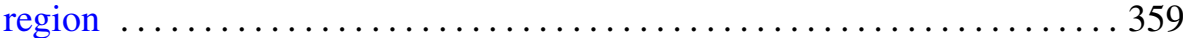

A. Ülger, Arens regularity sometimes implies the RNP 